\title{
Primordial germ cell development in zebrafish
}

\author{
Erez Raz
}

In sexually reproducing organisms, primordial germ cells (PGCs) give rise to gametes that are responsible for the development of a new organism in the next generation. These cells follow a characteristic developmental path that is manifested in specialized regulation of basic cell functions and behavior making them an attractive system for studying cell fate specification, differentiation and migration. This review summarizes studies aimed at understanding the development of this cell population in zebrafish and compares these results with those obtained in other model organisms.

Key words: primordial germ cells / PGG / cell migration / germ plasm / zebrafish

(C) 2002 Elsevier Science Ltd. All rights reserved.

\section{Introduction}

Several distinctive aspects of primordial germ cell (PGC) development make this cell population an appealing research subject in invertebrates and vertebrates. PGCs display a unique control over RNA transcription, exhibit a unique cell cycle program and show a characteristic cellular, as well as sub-cellular morphology that distinguish them from neighboring somatic cells. Considering that PGCs carry the genetic information to the next generation, the cells should maintain their totipotency, namely, the gametes that they form should be able to give rise to a complete new organism. This property of the germ cells is at the basis of studies aimed at understanding the molecular mechanisms that allow the germ cells to maintain a feature that is gradually lost in somatic cells. The ability of the germ cells to maintain totipotency is of

From the Germ Cell Development, Max-Planck-Institute for Biophysical Chemistry, Am Fassberg 11, 37070 Göttingen, Germany.

E-mail: eraz@gwdg.de

(C) 2002 Elsevier Science Ltd. All rights reserved.

1084-9521/02 / \$- see front matter particular practical importance for the purpose of modern genetic manipulation of different organisms. Finally, a common feature of PGCs is the fact that they migrate from the position where they are specified towards the position of the gonad where they differentiate into gametes. ${ }^{1-3}$ This behavior serves as a general model for long-range cell migration in multicellular organisms with the aim of identifying the underlying molecular mechanisms of this process. (for e.g. see References 4-7).

\section{Specification of primordial germ cells in zebrafish}

Germ-cell specification in fish was studied mainly in bony fish (teleosts) where the PGCs were recognized by their large size and by the typical electron dense 'nuage' structures that are found in germ cells of different organisms. These special cytoplasmic organelles contain specific RNAs and proteins and are thought to be important for germline development. Using these methodologies, the earliest time point at which germ cells were recognized in teleosts was around early somitogenesis stages. ${ }^{8-10}$ Thus, on the basis of these morphological criteria, PGCs could be identified only at a rather advanced stage of embryonic development. The question of the time and place at which these cells originate was therefore left open. The possibility remained that initially, a small population of PGCs existed and migrated to the position where it was identified morphologically. Nevertheless, the presence of electron dense material in the germ cells suggested that the mechanisms of PGC specification are common to fish and other germ plasm containing organisms such as Xenopus, Drosophila and Caenorhabditis elegans, where inheritance of asymmetrically localized cytoplasmic determinants, the germ plasm, is thought to direct cells to the germline lineage.

The study of fish germline development was revolutionized upon identifying the first molecular marker for cells of this lineage that is, the RNA of the zebrafish 
vasa gene, ${ }^{11,12}$ which encodes an ATP-dependent RNA helicase of the DEAD-box family. ${ }^{13,14}$ vasa was originally identified in Drosophila as a maternal effect gene required for the formation of abdominal segments and for germ-cell specification ${ }^{15}$ and is expressed in the germline of many species. ${ }^{16}$

Similar to many other organisms, zebrafish vasa mRNA is maternally supplied and it is detected throughout all stages of germline development. ${ }^{12,17-20}$ The cloning of the zebrafish vasa gene allowed close examination of germline cells from the earliest stages of embryonic development to later stages at which they differentiate into gametes. ${ }^{12,18,20}$ Whole-mount in situ hybridization at the 2-cell stage revealed that the vasa transcript is enriched at the marginal positions of the first cleavage plane. ${ }^{12}$ Similar accumulation of vasa transcript is found at the second cleavage plane leading to the formation of four stripes of intense vasa RNA staining (Figure 1, 4-cell stage). ${ }^{12}$ Mutant analysis and the use of microtubules-inhibiting drugs suggested that interaction between microtubules and vasa RNA-containing particles is essential for localization of the RNA at the correct position. ${ }^{21}$ Examination of the 3'-untranslated region (UTR) of the zebrafish vasa mRNA revealed four regions that are conserved among related teleosts (members of the ostariophysan clade that show similar localization of vasa mRNA). ${ }^{22}$ Indeed, analysis of $3^{\prime}$-UTR deletion constructs showed that a combination of one of these regions with other conserved regions was sufficient for the localization of the RNA in a pattern similar to the wild-type vasa RNA. ${ }^{22}$

Transmission electron microscopy showed that at the 4-cell stage vasa RNA is embedded in an electron dense matrix that resembles nuage, indicating that at this stage the RNA resides within the zebrafish germ plasm. ${ }^{20}$ During cleavage stages, up to the 1000-cell stage, no increase in the number of the vasa-containing cells is observed despite the dramatic increase in total cell number in the embryo during this period (Figure 1). The basis for this apparent discrepancy is that at these stages the germ plasm consists of a tight structure that is inherited only by one of the two dividing blastomeres, thereby keeping the number of vasa RNA-containing cells constant. ${ }^{20}$

From sphere stage on however, the tight morphology of the germ plasm changes as it spreads into the cytoplasm and is symmetrically distributed between the dividing blastomeres, thus resulting in a net increase in the cell number of vasa-positive PGCs (Figure 1). From about four cells at sphere stage (just before the initiation of zygotic vasa transcription), the cell number

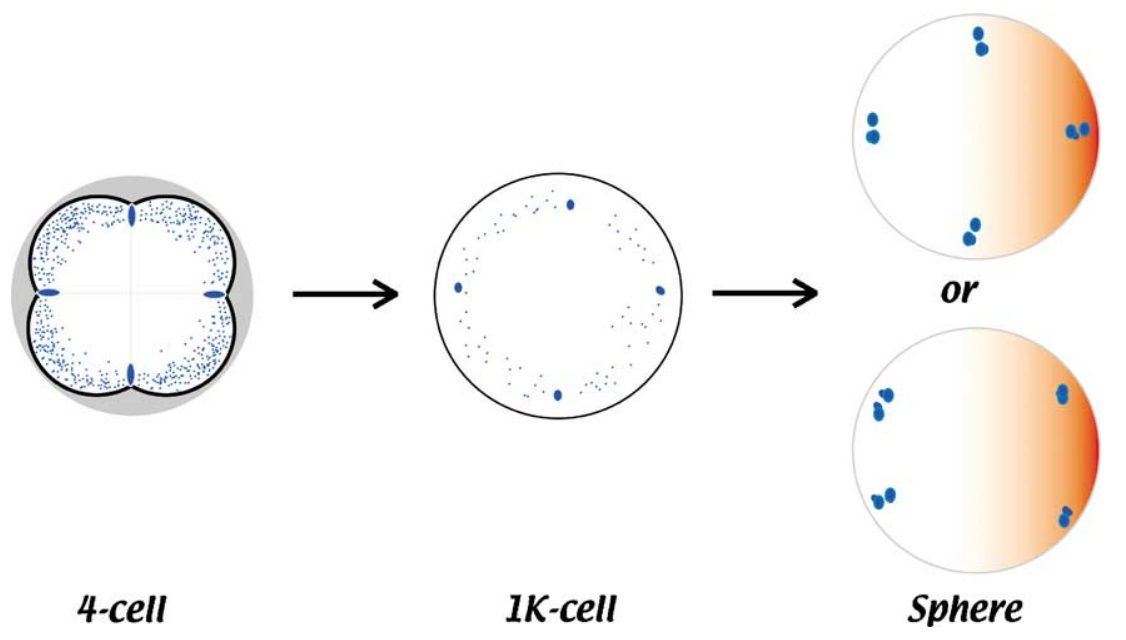

Figure 1. Specification of zebrafish PGCs by inheritance of germ plasm. At the 4-cell stage, the germ plasm containing vasa mRNA is enriched in the vicinity of the cleavage furrows (blue stripes). At early stages of development, vasa RNA and germ plasm can also be detected outside of the main four clusters (small blue dots), but this material is progressively degraded. During subsequent cell divisions, the four tight germ plasm structures segregate asymmetrically between the dividing cells thus, maintaining the number of germ plasm containing cells constant. At sphere stage, the germ plasm appears to spread in the cytoplasm and after cell division, it is inherited by both cells leading to an increase in the number of the PGCs. Considering that the orientation of the cleavage planes in zebrafish is random relative to the future dorsal aspect of the embryo, the four PGC clusters too, are found in random positions relative to the dorsal aspect of the embryo (red gradient at sphere stage). 
increases to $25-50$ cells by the end of the first day of development. $^{12,17,18,20}$

Interestingly, at the 4-cell stage vasa RNA is detected not only in the four main germ plasm clusters, but at a lower level along additional cleavage planes, as well and at later stages, in other cells around the margin $^{12,20,23}$ (Figure 1). Importantly, the RNA molecules which are not localized to the first two cleavage furrows and are not inherited by the PGCs, disappear shortly after. Additional RNA molecules, e.g. nanos 1 mRNA, exhibit similar early distribution of the maternally-supplied RNA that is subsequently refined to become PGC specific. ${ }^{24}$ This observation is similar to that described for RNA molecules of several genes important for germ cell development in Drosophila and in C. elegans that are degraded outside the germ cells. ${ }^{25,26}$ Analysis of cis-acting elements in the zebrafish vasa RNA revealed that sequences within the $3^{\prime}$-UTR, as well as within the coding portion of the RNA, are important for the differential stability of the RNA in the soma and the germ cells, resulting in PGC-specific distribution of the maternal RNA. ${ }^{23}$

One obvious obstacle in studying PGC development in zebrafish is that unlike the situation in Drosophila where a large number of genes involved in the development of these cells have been identified in maternal effect genetic screens, for a long while vasa was the only PGC marker in zebrafish. As an alternative for maternal effect genetic screens that are exceedingly arduous to carry out in zebrafish, ${ }^{27}$ 'molecular screens' for genes expressed in PGCs can be performed to identify molecules important for the PGC development. Such an approach brought about the identification of several genes that are expressed in the PGCs during early embryogenesis (for e.g. see References 24, 28; G. Weidinger and E. Raz, unpublished). Some of these genes correspond to genes whose function is important for germ cell development in other organisms, while others are novel genes or genes whose expression in the PGCs has not been previously described. An example of a gene isolated in this way is a nanos-related gene whose RNA is localized to the region of the germ plasm similar to vasa RNA. $^{24}$ nanos, an RNA binding protein, had been previously identified in Drosophila and in C. elegans as a germ plasm component. The shared morphological characteristics, as well as the identification of similar components (e.g. the gene products of vasa and nanos) in the germ plasm of invertebrates, anuran amphibians and zebrafish suggests that the mechanisms controlling early germ-cell development are conserved among these phylogenetically-distant organisms.

\section{PGG migration in zebrafish}

Similar to many other organisms, the PGCs in zebrafish are specified prior to the formation of the somatic portion of the gonad and at a different position. As described above, the four early clusters of PGCs are arranged at positions that are determined by the location of the first two cleavage planes (Figure 1). This mode of positioning the PGCs in zebrafish creates an interesting situation, wherein the PGCs initiate their migration from four different directions rather than from a defined point, as described for Drosophila, Xenopus and the mouse. A further complication stems from the fact that in zebrafish, the orientation of the first two cleavage planes is random relative to the position of the future dorsal aspect of the embryo. ${ }^{29,30}$ Therefore, zebrafish PGCs are initially found and specified at random positions with respect to the dorsoventral axis (Figure 1, red gradient at sphere stage), yet virtually all of them arrive at the target by the end of the first day of development ${ }^{12,17}$ (Figure 2).

Using vasa as a molecular marker for the migrating cells in combination with markers of somatic tissues yielded a detailed description of PGC migration in zebrafish (Figure 2). ${ }^{17}$ The analysis of PGC migration in wild-type zebrafish embryos showed that the process could be divided into discrete steps, which culminate in formation of two clusters by the end of the first day of development ${ }^{17}$ (Figure 2). An important intermediate step during the migration is evident by the end of gastrulation when the PGCs appear to align along the lateral and ventral borders of the trunk mesoderm, as well as along the border between the trunk and head mesoderm (Figure 2, 1-somite stage). Thus, the PGCs that are specified in marginal positions of the blastoderm are found in close proximity to the region where the mesoderm forms. This allows the cells to arrive at a common intermediate target during gastrulation despite the random dorsoventral starting position. Subsequent to PGC alignment along the mesoderm borders, during early somitogenesis, they migrate towards two lateral positions at the anteroposterior level of the first somites and form two PGC clusters (Figure 2, 8-somites stage). During later segmentation stages the PGC clusters migrate further posteriorly, to reach the level of the 8 th somite where their final target may reside ${ }^{12,17,31}$ (Figure 2, 16-somites stage) that is, cells comprising the somatic portion of gonad.

Similar to findings in other organisms, correct PGC migration in zebrafish appears to depend on cues provided by somatic cells. Specifically, the migrating cells appear to align along specific structures in the 


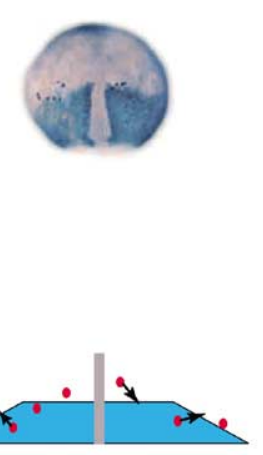

90\% Epiboly
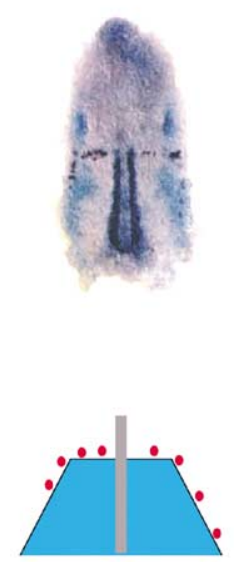

1-Somite
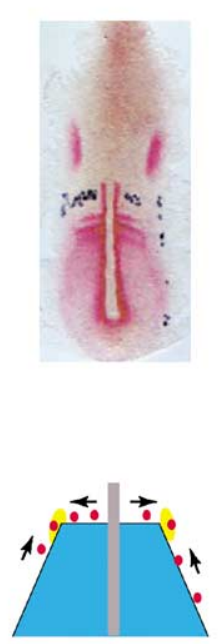

3-Somite
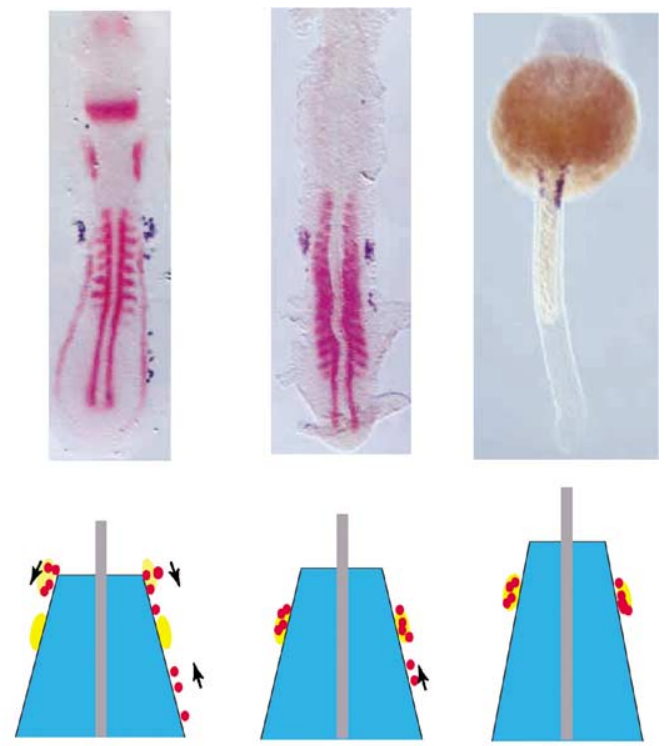

16-Somite

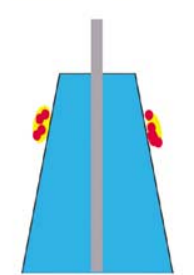

24 npf

Figure 2. Key steps in zebrafish PGC migration from gastrulation stages until the end of the first day of development. During gastrulation and early somitogenesis stages, the PGCs (red circles in schematic figures, black stained cells in the upper row) align along the lateral borders of the trunk mesoderm and between the head and trunk mesoderm (blue trapezoid in the schematic figures). After aligning along these structures, the PGCs start migrating towards two attraction points located at the level of the first three somites (yellow ellipsoids in the schematic figures) forming two bilateral-cell clusters by the 6- to 8-somite stage. In the following hours, the PGCs leave the first attraction point (pale yellow ellipsoid in the 8-somite stage) and migrate towards bilateral attraction points located around the level of the 10th somite (new yellow ellipsoid in schematic figures at 8-somites to $24 \mathrm{~h}$ post fertilizations (hpf)). This later site, where all PGCs are found by the end of the first day of development appears to be the position where the gonad develops during the following weeks. Reproduced from Reference 17 with permission from the Company of Biologists.

early embryo, they consistently avoid certain tissues and they migrate towards attraction points at defined positions within the developing embryo. A direct demonstration of somatic cell control of PGC migration was provided when the migration was analyzed in zebrafish mutants in which patterning and/or differentiation of specific somatic tissues was defective. ${ }^{17,31}$ This analysis showed that PGCs indeed respond to positional cues from somatic cells such that their absence results in aberrant PGC migration. ${ }^{17,31}$

Examining PGC migration in live embryos confirmed that these cells migrate actively towards the clustering position. ${ }^{22,31}$ Throughout their migration, the cells exhibit morphological features characteristic of motile cells, including extension of numerous cellular processes and a clear change of their position relative to their somatic neighbors. The migration patterns are highly dynamic: individual PGCs frequently alter their speed, direction and position relative to each other in the course of migration. These observa- tions suggest that the PGCs migrate as individual cells rather then as a coordinated group of cells.

\section{Maintenance of the fate of migrating PGC}

During their migration the PGCs face conflicting requirements regarding their response to cues provided by the somatic environment. On one hand, the PGCs should respond to signals provided by somatic tissues guiding them towards the gonad. On the other hand, while migrating, the PGCs should ignore signals that would lead to their differentiation into other cell types. Analysis of the fate of ectopic PGCs in zebrafish suggests that the migrating cells are committed to the PGC fate since they maintain the expression of molecular markers and retain characteristic PGC morphology even when residing in abnormal positions in the embryo. ${ }^{17}$ Thus, while they respond to the directional migration cues, being committed to the PGC fate, 
the cells do not respond to instructive differentiation cues.

In both Drosophila and C. elegans, the maintenance of the PGC fate, their survival and their incorporation into the gonad require the function of the nanos gene. ${ }^{32-36} \mathrm{~A}$ nanos-related gene that is expressed in PGCs has been identified in zebrafish (nanos $-^{24}$ ). Similar to the mRNAs of the Drosophila and C. elegans nanos genes, the zebrafish nanos 1 mRNA is provided maternally. The initial expression pattern of nanos 1 is comparable to that described for the zebrafish vasa mRNA which at early embryogenesis is found within an electron dense structure, the putative zebrafish germ plasm. ${ }^{12,20}$ During later blastula stages, nanos 1 mRNA is incorporated into the PGCs that characteristically migrate to form two PGC clusters. ${ }^{24}$ The mechanisms controlling the distribution of the maternal mRNA of nanos1 were found to be similar to those shown for vasa, in that the RNA is stabilized in the germ cells while being rapidly degraded in other cells in a process that depends on the 3 '-UTR of the gene. The function of nanos in germ cell development in zebrafish was determined by specifically inhibiting the translation of the corresponding RNA using morpholino antisense oligonucleotides. ${ }^{24,}{ }^{37}$ Interestingly, even when a very high amount of nanos 1 morpholinos was injected into the embryos no change in PGC numbers at pregastrulation stages was observed. However, while reducing nanos1 level did not affect PGC formation, it did deleteriously affect their migration and survival; In morpholino-injected embryos the PGCs failed to migrate normally, reached ectopic positions and exhibited morphological characteristics of cells undergoing programmed cell death. ${ }^{24}$ This phenotype is strikingly reminiscent of the nanos loss-of-function phenotype in Drosophila and C. elegans, which is manifested by cell survival and cell migration defects.

\section{PGC development in zebrafish as compared with that in other organisms}

The current understanding of PGC development in zebrafish suggests that the developmental program of these cells is controlled in a similar manner to that described for Drosophila, C. elegans and Xenopus and differs from that of mammals where inductive processes are responsible for PGC specification. ${ }^{1-3} \mathrm{~A}$ common denominator for the former group of organisms is that the PGCs there appear to be specified in sites containing specific asymmetrically localized cytoplasmic structures that can be visualized by electron microscopy - the germ plasm. Importantly, the identity and the function of germ plasm determinants appear to be conserved among these organisms. Moreover, the molecular mechanisms localizing specific RNAs to the germ plasm appear to be conserved between zebrafish and Xenopus ${ }^{22}$ while similar mechanisms controlling the stability and translatability of RNAs expressed in the PGCs are shared by Drosophila, C. elegans and zebrafish. ${ }^{24}$

Nevertheless, a closer look at germ cell development in zebrafish reveals some important differences between this vertebrate model organism and the invertebrates mentioned above. The most striking difference between the function of zebrafish germ plasm and that of Caenorhabditis and Drosophila concerns the time at which zygotic transcription is initiated in the germ cells. In both Caenorhabditis and Drosophila, the germ plasm represses early zygotic transcription. ${ }^{38-41}$ In contrast, in zebrafish, transcription appears to initiate in all nuclei at the same time, irrespective of the presence of germ plasm. ${ }^{20}$ Additional differences include the uniform Vasa protein distribution in zebrafish prior to PGC specification as opposed to the early PGC-specific expression of the Vasa proteins in C. elegans and in D. melanogaster. Further, unlike in $C$. elegans and in D. melanogaster, in zebrafish it is vasa RNA and not its protein that is part of the germ plasm at early stages of PGC development. ${ }^{20}$

\section{Conclusions and future directions}

In recent years our understanding of germline development in zebrafish was significantly enhanced by the identification of genes that are expressed in the PGCs. The basic questions concerning the mode of zebrafish PGC specification and origin are now answered. The cloning of the zebrafish vasa gene and the analysis of its expression pattern supports the notion that germ plasm is responsible for the early segregation and specification of the germline in fish. These findings, combined with the recently described function and regulation of the zebrafish nanos, revealed a previously unsuspected conservation between PGC development in invertebrates and fish. Considering that germ plasm was identified in other vertebrate groups such as the anuran amphibians and birds, it is conceivable that the finding regarding PGC development in fish would be relevant for those organisms, as well. ${ }^{1,42-44}$ While the mechanisms underlying PGC specification and early development 
differ between mammals and the species mentioned above, germ plasm components are expressed in the germline of mammals at later stages of development and are important for gametogenesis. ${ }^{45-47}$ Therefore, the genes and the mechanisms controlling early PGC development in zebrafish are likely to be relevant for germ cell development in mammals as well.

\section{Acknowledgements}

I am grateful to Michal Reichman for comments on the manuscript. The work in my lab is supported by grants from the DFG and the Volkswagen-Stiftung.

\section{References}

1. Saffman E, Lasko P (1999) Germline development in vertebrates and invertebrates. Cell Mol Life Sci 55:1141-1163

2. Wylie C (1999) Germ cells. Cell 96:165-174

3. Wylie C (2000) Germ cells. Curr Opin Genet Dev 10:410-413

4. Anderson R, Copeland Tk, Scholer H, Heasman J, Wylie C (2000) The onset of germ cell migration in the mouse embryo. Mech Dev 91:61-68

5. Howard K (1998) Organogenesis: Drosophila goes gonadal. Curr Biol 8:R415-R417

6. Moore La, Broihier Ht, Van Doren M, Lunsford Lb, Lehmann R (1998) Identification of genes controlling germ cell migration and embryonic gonad formation in Drosophila. Development 125:667-678

7. Van Doren M, Brohier Ht, Moore La, Lehmann R (1998) HMG-CoA reductase guides migrating primordial germ cells. Nature 396:466-469

8. Gevers P, Dulos J, Schipper H, Timmermans L (1992) Origin of primordial germ cells, as characterized by the presence of nuage, in embryos of the teleost fish Barbus conchonius. Eur J Morphol 30:195-204

9. Hamaguchi S (1982) A light- and electron-microscopic study on the migration of primordial germ cells in the teleost, Oryzias latipes. Cell Tissue Res 227:139-151

10. Timmermans L, Taverne N (1989) Segragation of primordial germ cells: their numbers and fate during early development of Barbus conchonius (Cyprinidae, Teleosti) as indicated by ${ }^{3} \mathrm{H}$-thymidine incorporation. J Morphol 202:225-237

11. Olsen LC, Aasland R, Fjose A (1997) A vasa-like gene in zebrafish identifies putative primordial germ cells. Mech Dev 66:95-105

12. Yoon C, Kawakami K, Hopkins N (1997) Zebrafish vasa homologue RNA is localized to the cleavage planes of 2- and 4-cell-stage embryos and is expressed in the primordial germ cells. Development 124:3157-3165

13. Hay B, Jan L, Jan Y (1988) A protein component of Drosophila polar granules is encoded by vasa and has extensive sequence similarity to ATP-dependent helicases. Cell 55:577-587

14. Lasko P, Ashburner M (1988) The product of the Drosophila gene vasa is very similar to eukaryotic initiation factor-4A. 335:611-617
15. Schupbach T, Wieschaus E (1986) Maternal-effect mutations altering the anterior-posterior pattern of the Drosophila embryo. Roux's Arch Dev Biol 195:302-317

16. Raz E (2000) The function and regulation of vasa-like genes in germ-cell development. Genome Biol 1:1017.1-1017.6

17. Weidinger G, Wolke U, Koprunner M, Klinger M, Raz E (1999) Identification of tissues and patterning events required for distinct steps in early migration of zebrafish primordial germ cells. Development 126:5295-5307

18. Braat A, Zandbergen T, Van De Water S, Goos H, Zivkovic D (1999) Characterization of zebrafish primordial germ cells: morphology and early distribution of vasa RNA. Dev Dyn 216:153-167

19. Braat Ak, Van De Water S, Goos H, Bogerd J, Zivkovic D (2000) Vasa protein expression and localization in the zebrafish. Mech Dev 95:271-274

20. Knaut H, Pelegri F, Bohmann K, Schwarz H, Nusslein-Volhard C (2000) Zebrafish vasa RNA but not its protein is a component of the germ plasm and segregates asymmetrically before germline specification. J Cell Biol 149:875-888

21. Pelegri F, Knaut H, Maischein H, Schulte-Merker S, NussleinVolhard C (1999) A mutation in the zebrafish maternal-effect gene nebel affects furrow formation and vasa RNA localization. Curr Biol 9:1431-1440

22. Knaut H, Steinbeisser H, Schwarz H, Nusslein-Volhard C (2002) An evolutionary conserved region in the vasa $3^{\prime}$ UTR targets RNA translation to the germ cells in the zebrafish. Curr Biol 12:454-466

23. Wolke U, Weidinger G, Köprunner M, Raz E (2002) Multiple levels of post-transcriptional control lead to germ line specific gene expression in the zebrafish. Curr Biol 12:289-294

24. Köprunner M, Thisse C, Thisse B, Raz E (2001) A zebrafish nanos-related gene is essential for the development of primordial germ cells. Genes Dev 15:2877-2885

25. Seydoux G, Fire A (1994) Soma-germline asymmetry in the distributions of embryonic RNAs in Caenorhabditis elegans. Development 120:2823-2834

26. Bashirullah A, Halsell S, Cooperstock R, Kloc M, Karaiskakis A, Fisher W, Fu W, Hamilton J, Etkin L, Lipshitz H (1999) Joint action of two RNA degradation pathways controls the timing of maternal transcript elimination at the midblastula transition in Drosophila melanogaster. EMBO J 18:2610-2620

27. Pelegri F, Schulte-Merker S (1999) A gynogenesis-based screen for maternal-effect genes in the zebrafish Danio rerio. Methods Cell Biol 60:1-20

28. Müller K, Thisse C, Thisse B, Raz E (2002) Expression of a linker histone-like gene in the primordial germ cells in zebrafish. Mech Dev, in press

29. Abdelilah S, Solnica-Krezel L, Stainier D, Driever W (1994) Implications for dorsoventral axis determination from the zebrafish mutation janus. Nature 370:468-471

30. Helde Ka, Wilson Et, Cretekos Cj, Grunwald Dj (1994) Contribution of early cells to the fate map of the zebrafish gastrula. Science 265:517-520

31. Weidinger G, Wolke U, Koprunner M, Thisse C, Thisse B, Raz E (2002) Regulation of zebrafish primordial germ cell migration by attraction towards an intermediate target. Development 129:25-36

32. Lehmann R, Nusslein-Volhard C (1991) The maternal gene nanos has a central role in posterior pattern formation of the Drosophila embryo. Development 112:679-691

33. Wang C, Lehmann R (1991) Nanos is the localized posterior determinant in Drosophila. Cell 66:637-647 
34. Wharton RP, Struhl G (1991) RNA regulatory elements mediate control of Drosophila body pattern by the posterior morphogen nanos. Cell 67:955-967

35. Subramaniam K, Seydoux G (1999) nos-1 and nos-2, two genes related to Drosophila nanos, regulate primordial germ cell development and survival in Caenorhabditis elegans. Development 126:4861-4871

36. Deshpande G, Calhoun G, Yanowitz Jl, Schedl PD (1999) Novel functions of nanos in downregulating mitosis and transcription during the development of the Drosophila germline. Cell 99:271-281

37. Nasevicius A, Ekker Sc (2000) Effective targeted gene knockdown in zebrafish. Nat Genet 26:216-220

38. Zalokar M (1976) Autoradiographic study of protein and RNA formation during early development of Drosophila eggs. Dev Biol 49:425-437

39. Seydoux G, Strome S (1999) Launching the germline in Caenorhabditis elegans: regulation of gene expression in early germ cells. Development 126:3275-3283

40. Asaoka M, Sano H, Obara Y, Kobayashi S (1998) Maternal Nanos regulates zygotic gene expression in germline progenitors of Drosophila melanogaster. Mech Dev 78:153-158
41. Van Doren M, Williamson Al, Lehmann R (1998) Regulation of zygotic gene expression in Drosophila primordial germ cells. Curr Biol 8:243-246

42. Rongo C, Lehmann R (1996) Regulated synthesis, transport and assembly of the Drosophila germ plasm. Trends Genet 12:102109

43. Houston D, King M (2000) Germ plasm and molecular determinants of germ cell fate. Curr Top Dev Biol 50:155181

44. Tsunekawa N, Naito M, Sakai Y, Nishida T, Noce T (2000) Isolation of chicken vasa homolog gene and tracing the origin of primordial germ cells. Development 127:2741-2750

45. Eberhart Cg, Maines Jz, Wasserman Sa (1996) Meiotic cell cycle requirement for a fly homologue of human deleted in azoospermia. Nature 381:783-785

46. Houston DW, King ML (2000) A critical role for Xdazl, a germ plasm-localized RNA, in the differentiation of primordial germ cells in Xenopus. Development 127:447-456

47. Tanaka S, Toyooka Y, Akasu R, Katoh-Fukui Y, Nakahara Y, Suzuki R, Yokoyama M, Noce T (2000) The mouse homolog of Drosophila Vasa is required for the development of male germ cells. Genes Dev 14:841-853 